\title{
Téoros
}

Revue de recherche en tourisme

\section{Ambiance des restaurants et expériences touristiques}

\section{François Pageau}

Volume 25, numéro 1, printemps 2006

Entre la culture du goût et le goût de la culture

URI : https://id.erudit.org/iderudit/1071034ar

DOI : https://doi.org/10.7202/1071034ar

Aller au sommaire du numéro

Éditeur(s)

Université du Québec à Montréal

ISSN

0712-8657 (imprimé)

1923-2705 (numérique)

Découvrir la revue

Citer cet article

Pageau, F. (2006). Ambiance des restaurants et expériences touristiques.

Téoros, 25(1), 43-49. https://doi.org/10.7202/1071034ar d'utilisation que vous pouvez consulter en ligne.

https://apropos.erudit.org/fr/usagers/politique-dutilisation/ 


\section{Ambiance des restaurants et expériences touristiques}

\section{François Pageau}

L'ouverture d'un nouveau restaurant représente évidemment un risque et, malgré tout, le succès est rarement prévisible. Plusieurs facteurs sont déterminants dans la réussite, soit la localisation commerciale, le marketing mix, la mise en marché et la campagne de lancement, mais aussi et surtout la qualité de la nourriture, la qualité du service et l'aménagement physique, dont le décor et l'ambiance. Ces éléments auront un impact non seulement sur la satisfaction de la clientèle, mais également sur la capacité du restaurant à attirer des segments de clientèles différents (Zeithmal et Bitner, 2003 : 297).

Comme l'aménagement physique est une composante relativement fixe, le concepteur d'un nouveau restaurant devrait apporter un grand souci au décor et à l'ambiance et considérer que ces derniers devraient idéalement correspondre aux attentes d'une clientèle particulière, touristique par exemple, de manière à l'attirer efficacement.

L'expérience recherchée par un touriste diffère selon le moment de la journée, selon ses intérêts personnels, ses activités, son goût de la découverte et selon ses motifs et les bénéfices qu'il recherche en fréquentant un restaurant particulier.

À certains égards, le Québec se positionne déjà très bien. Nombreux sont les établissements de qualité qui offrent une excellente nourriture dans un décor efficace et agréable. À titre d'exemple, le tiers des Américains adeptes du vin et des expériences culinaires qui traversent la frontière viennent au Québec (CCT, 2003 : 3). Les raisons qu'ils évoquent sont la réputation de la cuisine et des vins français ainsi que le cachet européen du Vieux-Québec et du Vieux-Montréal.
Pour le restaurateur, la cohérence entre ce que cherche le client et l'ambiance proposée par un établissement de restauration est fondamentale. L'ambiance proposée devient donc un élément stratégique de l'attraction d'un client. Les motifs et les bénéfices recherchés peuvent ainsi servir de base à une segmentation du marché et guider le choix d'un type d'ambiance approprié.

À l'échelle d'un marché national, comme un touriste ressentira des besoins variés durant son séjour, il importe, pour le satisfaire, d'assurer une diversité d'ambiances de restauration.

Cet article fait état d'une recherche exploratoire qui a modestement pour but de susciter la réflexion à propos de la segmentation des clientèles des restaurants du Québec, segmentation fondée sur quatre types de motifs en lien avec des ambiances caractéristiques des restaurants que l'on trouve plus particulièrement dans les villes de Montréal et de Québec.

\section{Méthodologie}

Cette recherche, de nature exploratoire, a été réalisée à partir de répertoires de restaurants et de visites d'établissements, de l'analyse de photographies et d'une synthèse d'écrits sur la segmentation de marché et les entreprises de services, notamment à l'égard des bénéfices recherchés.

À la suite de plusieurs interventions en entreprises et de plusieurs années d'enseignement de la gestion et de l'aménagement spécifiquement adaptés à la restauration, nous avons ressenti le besoin de mener cette étude dans le but de proposer une segmentation pertinente pour l'industrie de la restauration québécoise. Le concept "d'économie d'expérience », plus précisément dans sa dimension liée à l'ambiance et au décor, méritait, selon nous, d'être exploité en rapport à la segmentation du marché, afin de guider les concepteurs de nouveaux restaurants.

Dans un premier temps, nous avons effectué une révision des écrits sur la segmentation en restauration, d'où est né un cadre théorique pour segmenter autrement les clientèles des restaurants québécois. Dans un second temps, nous avons procédé à un examen de différents aménagements proposés dans les restaurants des villes de Montréal et de Québec, dans le but de les regrouper sous un minimum de vocables.

L'appellation des différents segments de marché et des types d'aménagement se base sur une approche qualitative et intuitive. Les dénominations choisies visent essentiellement à faire ressortir des qualités dominantes parmi les caractéristiques constatées.

Les limites de cette méthodologie résident dans l'effort de généralisation qu'exigeait l'identification de segments de marché et de types d'aménagement s'appliquant au plus grand nombre de clients et d'établissements. Des études quantitatives seront nécessaires pour raffiner les liens proposés entre les segments identifiés et les types d'aménagement qualifiés dans cette recherche.

Reste aussi l'acceptation des appellations des segments et des types d'ambiance par l'industrie, qui seront accueillies favorablement selon l'utilité qu'ils pourront générer. Le futur nous indiquera leur pertinence et leur utilité. 


\section{Définition d'un concept de restaurant}

L'industrie de la restauration québécoise est caractérisée par la présence d'un grand nombre d'établissements très variés, habituellement de petite taille, qui combinent dans leur offre de produits/services des éléments tangibles - l'assiette, la nourriture et la boisson - et des éléments intangibles - le service, la valorisation et le dépaysement créé par le décor et l'ambiance. Les restaurants sont à la fois des usines de production à petite échelle et des espaces de service dans lesquels le client "prend place", intervient, contribue et influence le déroulement de sa propre expérience (Zeithmal et Bitner, 2003 : 299).

Cette dualité « usine - service » en rend la gestion très complexe et la définition du produit/service encore plus subtile. En raison de la petite taille des établissements indépendants et de leurs ressources limitées, force est d'admettre que le recours aux études de marché dans le but de définir les paramètres du produit/service est plutôt exceptionnelle (Hafsi et al., 2003 : 180). Les grandes chaînes de restauration y ont recours régulièrement. Le soin apporté au design n'est pourtant pas exclusif aux grandes chaînes de restaurant, puisque les petits établissements indépendants se distinguent souvent à cet égard.

Même si les études de marché se déroulent dans les règles de l'art pour déterminer les mets les plus populaires, les caractéristiques du marché et du marketing mix de la concurrence, le choix des décors et de l'ambiance reste une décision personnelle du restaurateur, inspiré ou supporté quelquefois par un designer spécialisé. Le choix d'un type de décor est davantage une question de style, de mode, d'inspiration et de flair propres à l'artiste. Le fameux Concours Design Montréal ${ }^{1}$ a maintes fois démontré la pertinence de recourir à des professionnels du design dans un tel contexte. Par contre, comme le but poursuivi de l'aménagement est aussi souvent de surprendre le client, il est contradictoire de lui demander d'exprimer ses attentes pour s'y conformer.

Par ailleurs, les catégories traditionnelles (Spears et Gregoire, 2004 : 10), qui permettent de classer les différents services alimentaires, sont inappropriées pour orienter les entrepreneurs et les gestionnaires en restauration dans la définition d'un décor qui soit conforme à un concept particulier de restauration. Ces appellations correspondent davantage à des modes de service, de distribution et aux exigences liées aux permis. Elles ne tiennent pas compte des motifs et des comportements de la clientèle, comme le démontre l'encadré.

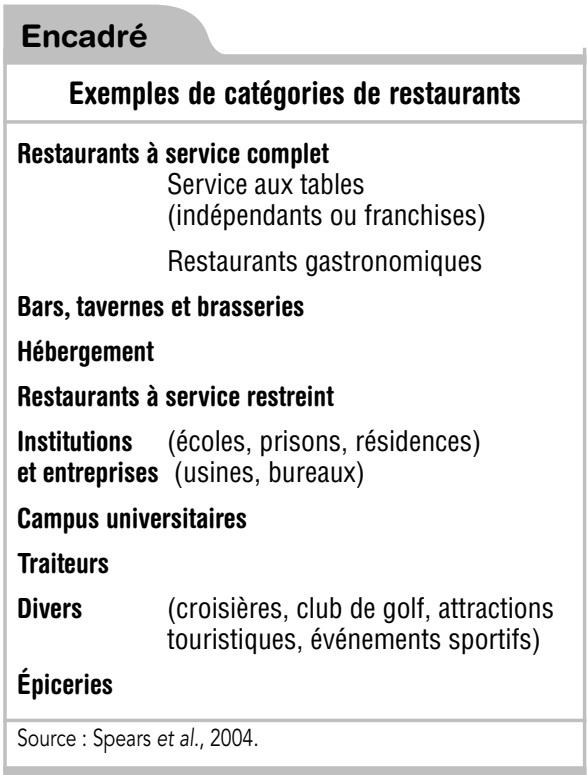

Si le bénéfice recherché par le client influence son choix et sa satisfaction, il nous apparaît alors intéressant d'essayer de fonder une autre classification qui serait plutôt basée sur les décors et les ambiances.

\section{Création d'une ambiance}

Pour concevoir leur établissement, les restaurateurs s'inspirent de ce qu'ils ont appris durant leur carrière, de ce qu'ils ont vu lors de leurs déplacements et de ce qui a remporté du succès. Ils utiliseront leur créativité, l'alignement stratégique sur les concurrents ou sur les concepts classiques et traditionnels (café, casse-croûte, bar, salle à manger, ligne de services, cafétéria, etc.). Ils s'interrogeront aussi sur des éléments du design, sans vraiment connaître les attentes réelles des clients qu'ils tentent d'attirer.

\section{Reflet d'une vision personnelle}

La définition du concept d'un nouveau restaurant indépendant, et plus spécifiquement de son décor et de son ambiance, est donc souvent le fruit d'une conception personnelle du restaurateur, idéalement en complicité avec un designer compétent et créatif, influencé par l'environnement, y compris par ce qu'il croit être les attentes de la clientèle et les tendances qui marquent l'époque.

\section{Reflet d'un marché}

Le concept d'un nouveau restaurant tient habituellement compte de son environnement immédiat, puisqu'il s'adressera d'abord à un marché local. À Montréal, sur les rues Saint-Laurent et Crescent par exemple, on trouve des restaurants très "tendance", soumis à des rénovations fréquentes de manière à se tenir à la mode et à attirer une clientèle «jet set » et de «foodies ». Les décors y sont habituellement très élaborés, à la mode et raffinés. Dans un style différent, les restaurants et les petites auberges de Baie-SaintPaul se fondent aussi sur l'environnement immédiat, mais, dans ce cas, pour refléter le calme et la tranquillité que recherchent les personnes qui fréquentent la région de Charlevoix. Les magnifiques hôtels Fairmount, héritage de CP Hôtels et du CN, qui inspirent quant à eux le raffinement et l'élégance, sont cohérents avec la recherche d'une expérience valorisante de villégiature.

\section{Reflet d'une époque}

Un nouveau restaurant est aussi le reflet de l'époque de sa création ainsi que de son propriétaire. Des restaurants icônes de Montréal tels que La Binerie, Schwart's, Da Giovanni, Ben's, Gibby's, Beauty's, Marvin's, Chez Milos, sont les témoins d'une autre époque, mais leur cachet et leur charme survivent au passage du temps. Les éléments de construction (murs, plafonds, planchers, éclairage, matériaux, etc.), la décoration (couleurs, papier peint, toiles, etc.), tout comme le mobilier, marquent l'époque de leur création.

L'ambiance d'un restaurant, résultat d'un ensemble d'éléments, allant du décor à l'éclairage, à la musique, en passant par l'uniforme et l'attitude des employés de service (Zeithmal et Bitner, 2003 : 299), est donc l'une des composantes qui contribuent directement à la satisfaction du client et qui caractérisent son produit/service.

\section{Construction d'une expérience forte}

Selon Pine Il et Gilmore, les « services » sont similaires à un spectacle et les restaurants ne sont autre chose que d'immenses scènes (Dumas, 2005). La nourriture représenterait même un second rôle dans la prestation et ce que le client achète serait fondamentalement l'expérience dont la nourriture fait partie (Pine II et Gilmore, 1998: 101). D'où l'importance de ne pas négliger les autres 
aspects et de rechercher la cohérence de l'ensemble des éléments qui composent l'expérience. Les installations physiques ont un rôle crucial car elles doivent créer l'environnement le plus crédible possible, tout en étant fonctionnelles et en facilitant le travail « des employés de contact » (Eigler et Langeard, 1987: 69). "Les services, en effet, ont besoin d'une mise en scène faisant appel à un décor et à un placement des acteurs. » (Idem : 68)

Cependant, les échecs des restaurants d'ambiance forte tels que Planet Hollywood, Red Lobster's et Movenpick, au Québec, malgré leur succès en Ontario, aux ÉtatsUnis et en Europe, font réfléchir sur la place donnée au spectacle et la mise en scène. À l'inverse, le succès de La Cage aux Sports illustre fort bien le principe.

\section{Limites des segmentations actuelles}

L'importance de l'ambiance étant établie, qu'en est-il des catégorisations des clientèles qui pourraient y être associées ? En premier lieu, il est important de noter que nos recherches démontrent qu'il n'existe pas de segmentation du marché concertée et reconnue dans l'industrie et qui serait une base utile pour tous.

En second lieu, nous allons examiner trois types de segmentation actuels. Une première que l'on pourrait appeler les appellations courantes pour catégoriser les clientèles, une deuxième selon les caractères démographiques et une troisième spécifiquement axée sur le touriste.

\section{Appellations courantes}

La segmentation de la clientèle des restaurants du Québec est difficile à définir, car elle est très rarement homogène et commune à l'ensemble des établissements. Les différents types de clientèles sont souvent appelés comme suit dans l'industrie :

- clientèle d'affaires: cols blancs (hommes et femmes, avec ou sans compte de dépenses);

- clientèle de travailleurs cols bleus;

- clientèle de familles et d'amis (touristes et locaux) ;

- clientèle de couples (touristes et locaux) ;

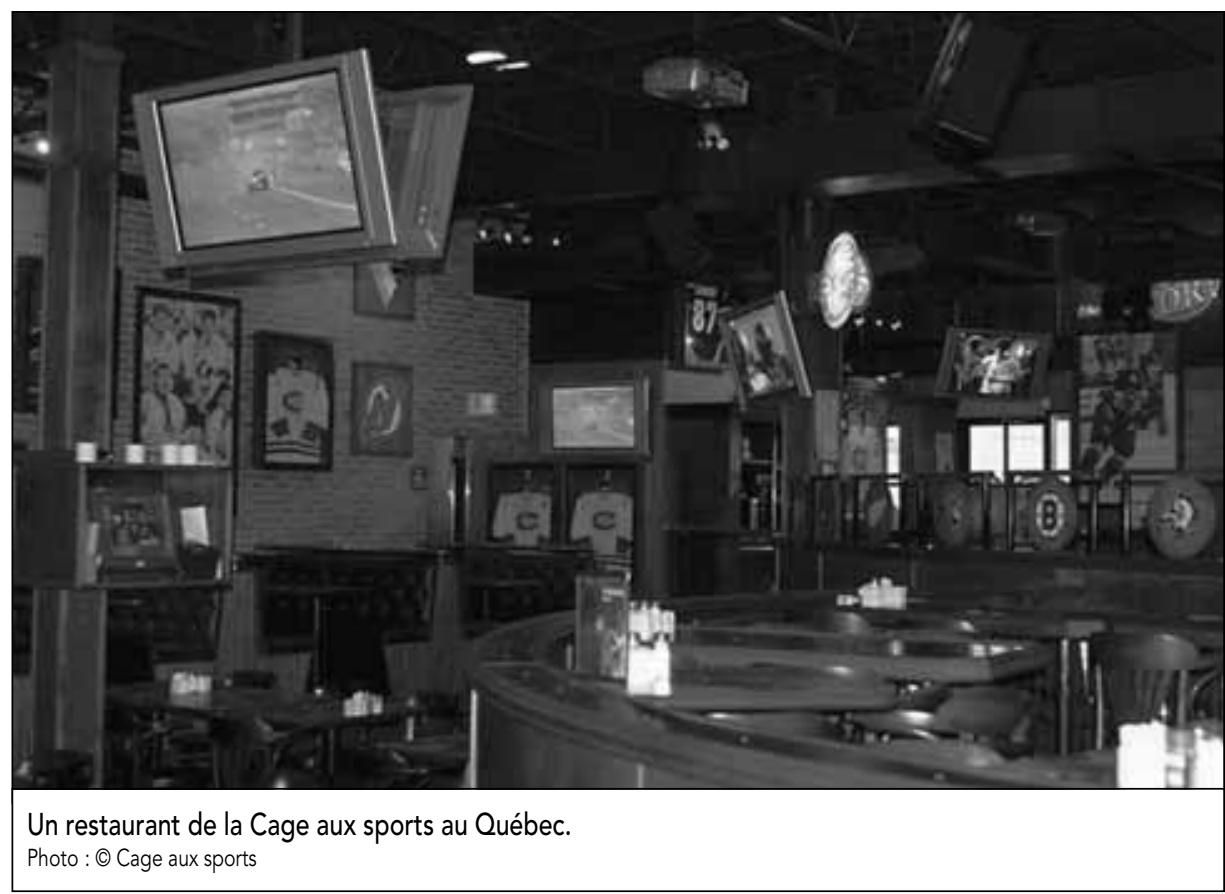

- clientèle de congressistes;

- clientèle de touristes (solo, couples, familles, groupes);

- clientèle de foodies, passionnés et amateurs ;

- hommes, femmes, jeunes et adolescents ;

- clientèle de personnes qui assistent à un divertissement (cinéma, festival, théâtre, spectacle, concert, etc.) ;

- clientèle de voyageurs (camions, autobus, voitures, avions, trains, bateaux).

L'avantage de ces appellations et que leur compréhension est aisée et relativement facile à attribuer au client qui pénètre dans un établissement.

Leur désavantage réside dans la difficulté d'en déduire des besoins et des attentes précis. De plus, ces appellations sont hétérogènes, ne sont pas mutuellement exclusives et la même personne peut se voir qualifiée dans plusieurs appellations en même temps.

\section{Caractères démographiques}

La clientèle des restaurants est rarement homogène et le même individu peut fréquenter différents types de restaurants. Les caractères démographiques sont donc insuffisants pour réfléchir de manière utile sur la catégorisation des restaurants.

\section{Touristes}

Durant son séjour, un même touriste voudra fréquenter différents types de restaurants, par exemple le congressiste qui voudra fréquenter un petit resto typique de la région, participer aux banquets prévus au congrès et fréquenter les comptoirs rapides dispersés autour du centre des congrès. Le même individu ressentira donc des besoins différents pendant son séjour. En conséquence, aucun établissement, malgré la constance des caractéristiques démographiques du congressiste, ne pourra prétendre être en mesure de répondre à tous les besoins qu'il ressentira dans une même journée.

La CCT (Commission Canadienne du Tourisme), quant à elle, a défini plusieurs segments de marché tirés de variables de l'Enquête sur les activités et les préférences en matière de voyage (EAPV) (tableau 1). Dans ce cas spécifique, la CCT a créé une segmentation utile à l'étude de l'EAVP.

L'avantage de cette segmentation est sa facilité d'utilisation et la catégorisation univoque des individus. Elle favorise aussi la mise en marché et les efforts de publicité. Son utilité est toutefois limitée à l'étude spécifique à laquelle elle est destinée. En ce sens, elle est difficilement transposable pour un restaurateur qui vise un marché plus large que les touristes d'agrément qui pratiquent une activité précise. 
Tableau 1

Segments de marché des voyageurs d'agrément au Canada, selon le but du voyage

\begin{tabular}{l|c|c} 
Activités et préférences en matière de voyage & Total & $\begin{array}{c}\text { Adeptes du vin et } \\
\text { des expériences culinaires }\end{array}$ \\
\hline Adultes (18+) & $\mathbf{2 6 , 3}$ millions & $\mathbf{5 , 5}$ millions \\
\hline Vin et expérience culinaire & 5,5 & 5,5 \\
Aventure de plein air douce & 7,1 & 1,9 \\
Aventure de plein air dure & 3,2 & 1,0 \\
Patrimoine & 8,3 & 3,1 \\
Arts de la scène & 3,8 & 1,7 \\
Arts visuels & 7,0 & 2,6 \\
Activités de plein air hivernales (à l'exclusion du ski) & 3,7 & 1,2 \\
Ski alpin & 4,6 & 1,3 \\
\hline
\end{tabular}

Source : CCT, 2003.

En somme, les classifications examinées ont d'évidentes limites et nous croyons que la segmentation d'un marché sur la base des motifs et des bénéfices recherchés représente une meilleure approche.

\section{Segmentation de marché, bénéfices recherchés et comportements}

Dans le but de segmenter le marché des services, les critères de situation sont plus pertinents et plus efficaces que les critères socioéconomiques (Eigler et Langeard, 1987 : 22). Par exemple, dans le secteur de l'hôtellerie, le motif du déplacement professionnel ou de loisir est un critère fondamental qui va déterminer en grande partie les attentes et les comportements d'un même individu.

Selon Reid et Bojanic (2006: 91), les segments de marché fondés sur le comportement se distinguent selon les besoins personnels, les motifs, l'expérience, la personnalité et l'image de soi, les perceptions et les attitudes. Ainsi, le comportement du client est animé par son besoin et la motivation qu'il ressent pour le satisfaire, le comportement qu'il imagine et qu'il applique pour obtenir satisfaction, ce qui peut servir de base pour les segmenter en différents groupes distincts.

L'avantage de cette segmentation basée sur les motifs et les bénéfices recherchés est la flexibilité qu'elle offre pour répondre aux besoins différents et ressentis par un même individu durant son séjour. Ainsi, qu'il soit homme ou femme, col blanc ou bleu, congressiste ou touriste avec un revenu élevé ou faible, il fréquentera un fast food le matin car il est pressé et recherche la sécurité, un bistro (dont le terme est issu du russe "bistra " qui veut dire "vite») le midi pour bien manger et être servi sans qu'il ait à y consacrer trop de temps et, finalement, un restaurant gastronomique en soirée pour la découverte et la valorisation personnelle qu'il en retire.

Les désavantages de cette segmentation résident donc dans la difficulté de mesurer le potentiel des segments basés sur les bénéfices recherchés à des fins d'étude et de recherche. Les caractères démographiques interfèrent également dans le choix que fera le client. Néanmoins, cette segmentation est utile pour le concepteur de restaurant dans sa définition des clientèles à satisfaire.

\section{Cohérence de l'ambiance avec la clientèle visée}

Puisque l'ambiance et le décor d'un restaurant possèdent un caractère d'esthétisme, il convient de les lier au besoin d'esthétisme de la clientèle, donc à ses attentes à cet égard. Pour le client, la salle à manger devient un environnement valorisant en termes d'image de soi. Un client voudra fréquenter un établissement qui correspond à ce qu'il est ou à ce qu'il voudrait être.

\section{Facteurs personnels}

Chaque consommateur a une personnalité unique et une image de soi. Nous avons donc regroupé les marchés selon différentes classifications de personnes qui vivent de certaines manières ou qui désirent s'associer à ceux qui le font: swingers, conservateurs, leaders et suiveurs (Reid et Bojanic, 2006 : 95).
Une étude de Kotler et al. (2003) présente les critères priorisés par la clientèle de trois types de restaurants (tableau 2). L'ambiance est utilisée à la fois comme critère de qualité et comme base de catégorisation.

Le processus de prise de décision du client repose sur des influences externes - telles que la culture, son niveau socioéconomique, ses groupes de référence et la composition de son ménage - et des facteurs internes - tels que ses besoins, ses expériences passées -, mais également sur sa personnalité et son image de soi ainsi que sur ses perceptions et ses attitudes (Reid et Bojanic, 2006 : 99).

Les attentes de la clientèle nord-américaine face aux entreprises de services incluent, depuis quelques décennies, la dimension «expérience ", comme l'ont démontré Pine II et Gilmore (1998). La restauration est particulièrement concernée par cette nouvelle dimension de l'expérience, puisque l'intérêt de la clientèle et l'accent du produit/service ne reposent plus uniquement sur l'assiette ou le service de l'assiette comme tels, mais aussi sur le décor, le scénario, les émotions, la thématique, le rôle des employés de contact, les renforcements sensoriels et l'intensité du dépaysement. Le restaurateur cherche ultimement à générer une clientèle régulière ou qui lui fera bonne presse et bonne critique et tous ces critères contribueront à atteindre cet objectif.

Reid et Bojanic (2006 : 123) arguent que les entreprises "must consider differences in lifestyle and consumer behaviour, all of which add special challenges to the marketing of the product-service mix for hospitality and tourism organizations ». Conséquemment, il est utile d'associer un comportement spé-

\section{Tableau 2}

Critères recherchés par les clients de restaurants

\begin{tabular}{l|c|c|c} 
& Populaire & D'ambiance & Gastronomique \\
\hline $\begin{array}{l}\text { Qualité de } \\
\text { la nourriture }\end{array}$ & & Priorité & Priorité \\
$\begin{array}{l}\text { Variété du } \\
\text { menu }\end{array}$ & Priorité & & \\
Prix & & \\
$\begin{array}{l}\text { Ambiance } \\
\text { Facteurs } \\
\text { pratiques } \\
\text { (convenience) }\end{array}$ & Priorité & & \\
\hline
\end{tabular}

Source : Kotler et al., $2003: 274$ 
cifique à un groupe de clients fidèles. Ces auteurs proposent par ailleurs une segmentation sur la base des bénéfices recherchés par la clientèle: "Market segmentation can be based on the benefits that consumers are seeking when they purchase a product." (Idem : 135) Une entreprise de restauration pourrait donc déterminer les bénéfices recherchés par sa clientèle et ensuite concevoir et adapter l'aménagement, le décor et l'ambiance en fonction des clientèles visées.

La segmentation comportementale consiste à regrouper les acheteurs selon les occasions d'achats, le statut d'utilisateur, le taux d'utilisation, la fidélité à la marque, la prédisposition à la marque, l'attitude à l'égard du service et les avantages recherchés, par exemple : l'économie (bas prix), la protection (sécurité), l'esthétisme et le goût (Kotler et al., 2003 : 274, Kotler et al., 2000 : 280).

\section{Ambiances identifiées}

Dans le contexte de nos travaux, nous avons identifié cinq principaux types de décor et d'ambiance. Ces cinq appellations sont issues des caractéristiques dominantes que nous avons identifiées en regroupant des restaurants qui avaient des ambiances et des décors similaires : divertissement, folklorique, tendance, sympathique et accessible, hygiénique et démocratique, et luxe classique.
- L'ambiance " divertissement, folklorique ou typique " d'une région, laquelle regroupe les restaurants qui mettent en valeur un thème lié à l'histoire, à la culture, aux sports ou encore un pays étranger ou une destination exotique. Le décor et l'ambiance devraient transporter le client et lui faire croire qu'il se trouve dans autre lieu ou encore dans un lieu authentique de la région qu'il visite.

- L'ambiance " tendance, haut de gamme moderne » réunit les restaurants qui font référence aux nouveaux riches, très tendance, modernes et sensuels. L'accent est mis sur un design poussé et audacieux, souvent associé à un designer de renom, un jeune chef vedette ou une personnalité publique.

- Les restaurants "sympathiques et accessibles » sont des restaurants très bien aménagés et décorés, mais qui n'intimident aucunement les différents groupes de la société. On y met l'accent sur le confort et l'accueil et davantage sur un design populaire et prudent.

- Les restaurants "hygiéniques et démocratiques " sont habituellement dénudés et décorés sobrement, mais avec goût. Reflet de la culture nord-américaine; ce type d'ambiance est résistant, facile d'entretien, prudent, sobre et flexible. Polyvalents et très pratiques, ces restau-

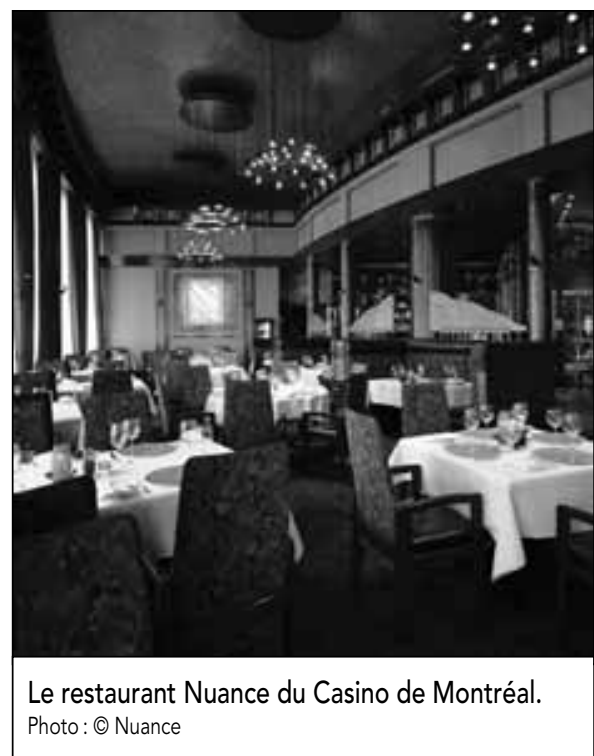

rants procurent sécurité et contrôle à tous les types de clientèles.

- L'ambiance «luxe classique » est le reflet du conservatisme d'affaires, souvent traditionnel, quelquefois moderne; le design est élaboré, il valorise et réconforte la clientèle habituellement aisée à la recherche de prestige et de valorisation personnelle.

Ces ambiances, appliquées aux restaurants du Québec, sont illustrées au tableau 3. Notons que les catégories permettent aussi

Tableau 3

\begin{tabular}{|c|c|c|c|c|c|}
\hline \multicolumn{6}{|c|}{ Types d'ambiance } \\
\hline $\begin{array}{l}\text { Type } \\
\text { d'ambiance }\end{array}$ & $\begin{array}{l}\text { Divertissement, } \\
\text { folklorique } \\
\text { ou typique } \\
\text { d'une région }\end{array}$ & $\begin{array}{l}\text { «Tendance ", } \\
\text { haut de gamme } \\
\text { moderne } \\
\text { (à la mode) }\end{array}$ & $\begin{array}{l}\text { Sympathique } \\
\text { et accessible }\end{array}$ & $\begin{array}{l}\text { Hygiénique et } \\
\text { démocratique }\end{array}$ & Luxe classique \\
\hline $\begin{array}{l}\text { Éléments } \\
\text { d'ambiance }\end{array}$ & $\begin{array}{l}\text { Décor élaboré } \\
\text { Musique populaire } \\
\text { Personnel costumé } \\
\text { Salle animée }\end{array}$ & $\begin{array}{c}\text { Décor et mobilier modernes } \\
\text { Ambiance feutrée } \\
\text { Éclairage tamisé } \\
\text { Musique lounge } \\
\text { Matériaux nouveaux } \\
\text { Designer reconnu }\end{array}$ & $\begin{array}{c}\text { Décor simple et de bon goût } \\
\text { Mobilier sobre } \\
\text { Intimité } \\
\text { Éclairage variable } \\
\text { Musique populaire et douce }\end{array}$ & $\begin{array}{c}\text { Décor simple } \\
\text { Tables et chaises faciles à laver } \\
\text { Couleurs éclatantes } \\
\text { Poubelles près des portes } \\
\text { Journal gratuit } \\
\text { Menus visibles }\end{array}$ & $\begin{array}{c}\text { Décor élaboré } \\
\text { Musique classique } \\
\text { Personnel professionnel } \\
\text { Matériaux nobles }\end{array}$ \\
\hline Exemples & $\begin{array}{c}\text { Cabanes à sucre } \\
\text { Restaurants à thème } \\
\text { L'Astral } \\
\text { La Binerie } \\
\text { Hard Rock Café } \\
\text { La Roche pleureuse } \\
\text { La Cage aux Sports } \\
\text { La Maison hantée } \\
\text { Dragon Rouge }\end{array}$ & $\begin{array}{c}\text { Restaurants tendances } \\
\text { Toqué ! } \\
\text { Newtown } \\
\text { Le Bistango } \\
\text { Queue de Cheval }\end{array}$ & $\begin{array}{c}\text { Service complet, } \\
\text { franchises ou non } \\
\text { St-Hubert } \\
\text { Amir } \\
\text { Commensal } \\
\text { Second Cup } \\
\text { Pacini }\end{array}$ & $\begin{array}{c}\text { Franchises } \\
\text { McDonald's } \\
\text { Tim Horton's } \\
\text { La Belle Province } \\
\text { Subway }\end{array}$ & $\begin{array}{c}\text { Beaver Club } \\
\text { Mount Stephen Club } \\
\text { Nuance } \\
\text { Baccarat }\end{array}$ \\
\hline
\end{tabular}

Note: Les exemples sont donnés afin d'illustrer nos propos et ne représentent pas une liste exhaustive. Les établissements nommés le sont en référence avec la vision personnelle de l'auteur au moment d'écrire ces lignes (janvier 2006). 


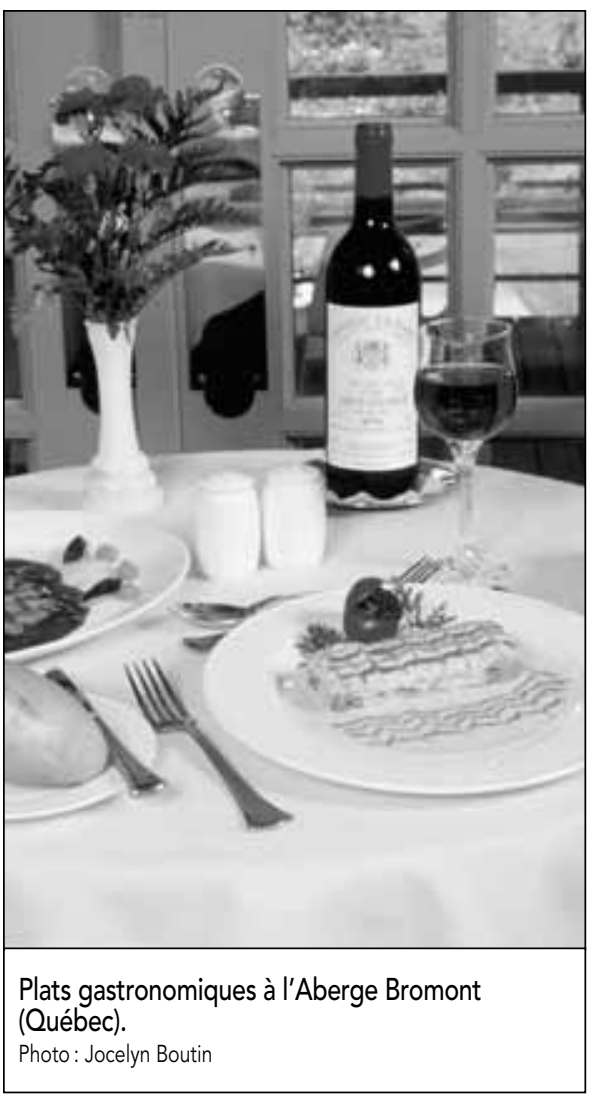

d'évoquer d'autres éléments du produit/service tels que la nourriture et le service, qui correspondent de manière cohérente aux attentes de la clientèle.

De cette segmentation basée sur les bénéfices recherchés par la clientèle découle l'identification de cinq types de clientèles :

- Le «spectateur découvreur » est le touriste curieux en quête de nouvelles expériences et de divertissement. II est à la recherche de souvenirs, de références et de connaissances qui enrichiront son séjour.

- L' « hédoniste tendance » est le touriste jet set, à la recherche de différents plaisirs et désireux de maintenir son image et son style innovateur et branché.

- Le « décontracté prudent » est le touriste détendu, assez conciliant, mais qui prend des risques calculés. II ne veut pas trop de protocole, mais recherche un certain confort et un niveau de prestation en fonction du prix.

- Le «pressé autonome » est le touriste qui a peu de temps pour manger et qui, entre deux activités, cherche à manger à bon compte, dans un environnement sécuritaire, propre et connu, dans lequel il gardera le contrôle sur le temps de service et la méthode de paiement.

- L'«aristocrate» est le touriste sophistiqué, souvent habitué à fréquenter les palaces et les endroits réservés aux plus nantis et qui recherche la valorisation et le summum en termes de produits et services.

En ayant en tête le lien qui peut exister entre le motif du client et l'ambiance recherchée, le restaurateur aura avantage à adapter le décor et l'aménagement en fonction du ou des segments de clientèles qu'il vise. Bien plus encore, il sera ainsi en mesure de composer un marketing mix et créer une expérience client qui correspondent aux attentes et aux motifs qui animent particulièrement le client lorsque celui-ci se présente chez lui.

Le tableau 4 propose une description sommaire de ces segments de clientèles et des ambiances qui s'adressent à chacun. Le restaurateur pourra s'en inspirer pour créer l'ambiance appropriée. Les détails du décor et du design restent à définir afin de donner la couleur propre et spéciale de l'établissement.

Tableau 4

Segmentation de la clientèle

\begin{tabular}{|c|c|c|c|c|c|}
\hline Segment & Spectateur découvreur & Hédoniste tendance & Décontracté prudent & Pressé autonome & Aristocrate \\
\hline $\begin{array}{l}\text { Bénéfices } \\
\text { recherchés }\end{array}$ & $\begin{array}{c}\text { Découverte } \\
\text { Expérience approfondie } \\
\text { Animation } \\
\text { Souvenir }\end{array}$ & $\begin{array}{c}\text { Hédonisme } \\
\text { Plaisir des sens } \\
\text { Esthétisme } \\
\text { Gastronomie } \\
\text { Matériaux nouveaux } \\
\text { Designer reconnu } \\
\text { But: impressionner, } \\
\text { séduire, image de soi }\end{array}$ & $\begin{array}{c}\text { Confort } \\
\text { Calme et assurance } \\
\text { Gastronomie bon marché }\end{array}$ & $\begin{array}{c}\text { Utilitarisme } \\
\text { Rapidité } \\
\text { Bon prix } \\
\text { Produit/service prévisible } \\
\begin{array}{c}\text { But : contrôler le rythme, } \\
\text { intervenir }\end{array}\end{array}$ & $\begin{array}{c}\text { Prestige et } \\
\text { reconnaissance sociale } \\
\text { Esthétisme } \\
\text { Conservatisme } \\
\text { Sécurité et } \\
\text { valorisation personnelle } \\
\text { But: impressionner, } \\
\text { sécuriser }\end{array}$ \\
\hline $\begin{array}{l}\text { Échelle des } \\
\text { besoins de } \\
\text { Maslow }\end{array}$ & $\begin{array}{l}\text { Estime de soi } \\
\text { Réalisation de soi }\end{array}$ & $\begin{array}{c}\text { Appartenance } \\
\text { Estime de soi } \\
\text { Réalisation de soi }\end{array}$ & $\begin{array}{l}\text { Appartenance } \\
\text { Estime de soi }\end{array}$ & $\begin{array}{l}\text { Besoins primaires } \\
\text { Sécurité }\end{array}$ & $\begin{array}{l}\text { Estime de soi } \\
\text { Réalisation de soi }\end{array}$ \\
\hline $\begin{array}{l}\text { Type } \\
\text { d'ambiance }\end{array}$ & $\begin{array}{l}\text { Divertissement, } \\
\text { folklorique ou typique } \\
\text { d'une région }\end{array}$ & $\begin{array}{l}\text { "Tendance ", } \\
\text { haut de gamme moderne } \\
\text { (à la mode) }\end{array}$ & Sympathique et accessible & Hygiénique et démocratique & Luxe classique \\
\hline \multirow[t]{2}{*}{$\begin{array}{l}\text { Expérience } \\
\text { recherchée }\end{array}$} & $\begin{array}{l}\text { Spectacle intense } \\
\text { et scénarisé }\end{array}$ & $\begin{array}{l}\text { Sans tabou, avec un décor } \\
\text { «mode » et personnel de } \\
\text { contact stylé }\end{array}$ & Anticipée et décontractée & Pratique, prévisible, & $\begin{array}{l}\text { Service élaboré et méticuleux, } \\
\text { respect des règles de l'art, } \\
\text { décorum, médiateurs nombreux }\end{array}$ \\
\hline & $\begin{array}{l}\text { Axée sur les attraits, } \\
\text { l'histoire,le caractère } \\
\text { québécois,les sports, } \\
\text { un pays étranger ou } \\
\text { une destination exotique }\end{array}$ & $\begin{array}{l}\text { Axée sur les sens, la } \\
\text { découverte gastronomique, } \\
\text { la rareté des produits, } \\
\text { l'audace et l'image actuelle } \\
\text { et jet set de la clientèle } \\
\text { et du personnel }\end{array}$ & $\begin{array}{l}\text { Axée sur la valorisation et } \\
\text { l'implication du client, } \\
\text { accent sur une « valeur } \\
\text { perçue » optimalesimple et } \\
\text { sécurisante }\end{array}$ & $\begin{array}{l}\text { Axée sur le mode de vie } \\
\text { nord-américain, la } \\
\text { modernité, le service rapide } \\
\text { et courtois et souci de la } \\
\text { clientèle composée de } \\
\text { jeunes et de familles }\end{array}$ & $\begin{array}{l}\text { Axée sur l'appartenance } \\
\text { à un statut d'élite sociale, } \\
\text { souvent liée au monde des } \\
\text { affaires ou à l'aristocratie }\end{array}$ \\
\hline
\end{tabular}

Source : Données recueillies par les auteurs. 


\section{Conclusion}

Les attentes et les besoins ressentis par le client sont les moteurs de son comportement et de l'orientation de son choix quant au(x) restaurant(s) qu'il voudra fréquenter. Le désir de vivre une expérience particulière varie durant le séjour d'un touriste. II est donc différent selon le type de restaurant choisi. L'expérience et l'ambiance recherchées varieront en fonction des motifs du moment.

Si l'ambiance est clairement définie et anticipée, le client potentiel pourra choisir efficacement en fonction des bénéfices qu'il recherche, sans toutefois négliger l'importance de la qualité de la nourriture et du service. Plus son degré de satisfaction sera élevé, plus il aura une propension à retourner à l'établissement et à le recommander.

Les limites de la classification proposée résident dans la difficulté de quantifier et l'impossibilité de suivre l'évolution des clientèles. Évidemment cette recherche est exploratoire et des études plus rigoureuses pourraient éventuellement contredire, valider ou nuancer les différents types d'ambiance, les segments de clientèles proposés et les liens que nous avons avancés entre eux. II reste néanmoins que chaque type d'ambiance de

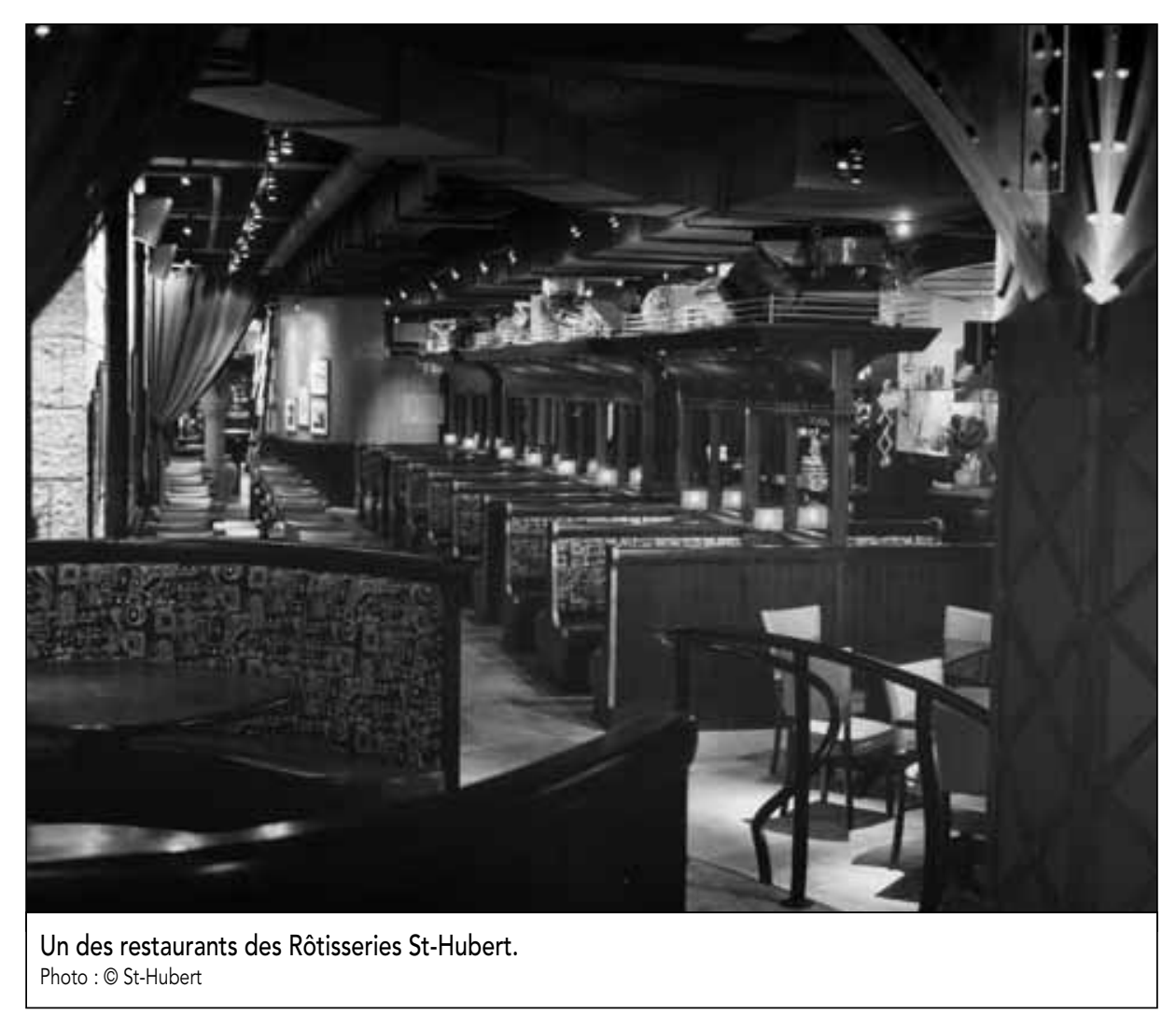

restaurant a sa raison d'être et est susceptible de répondre aux divers motifs et besoins ressentis par les touristes durant leur séjour.

Pour les organismes qui s'engagent dans une réflexion de définition de produit touristique comme «Réinventer Montréal », qui vise à stimuler l'imaginaire et à susciter des idées et des solutions mobilisatrices pour que la ville soit créative, humaine, propre, festive, diverse, équilibrée, prospère et généreuse, la préoccupation accordée au design des établissements de restauration sera pertinente puisque que celui-ci constitue un élément visible et palpable pour le touriste qui fréquentera la ville.

Des destinations touristiques importantes, comme la ville de Montréal et la ville de Québec, auraient avantage à offrir une diversité d'établissements de restauration qui couvrent une large gamme d'ambiances, de manière à répondre efficacement aux différents bénéfices recherchés par la clientèle touristique. Les restaurants de ces villes auront avantage à définir clairement leur décor et à faciliter l'identification de leur ambiance, de manière à satisfaire pleinement les touristes.
François Pageau est professeur et chargé de cours universitaire en gestion hôtelière et de la restauration à l'Institut de tourisme et d'hôtellerie du Québec. II enseigne le cours Stratégies des entreprises hôtelières et de la restauration.

\section{Notes}

1 Consulter à ce sujet [http://www.commerce designmontreal.com/].

\section{Bibliographie}

Bitner, J., et B. Booms (1981), « Marketing Strategies and Organizational Structures for Service Firms ", dans J. Donnelly et W. George (dir.) Marketing of services, American Marketing Association, Chicago.

CCT-Commission canadienne du tourisme (2003), "Touristes américains adeptes du vin et des expériences culinaires: Analyse spéciale de l'Enquête sur les activités et les préférences en matière de voyages (EAPV)", Research Resolutions \& Consulting Ltd., Ottawa, p. 41.

Dumas, Léonard (2005), « L'expérience hôtelière: Une valeur ajoutée", Revue HRI, Québec, vol. 9, n 5, septembre.

Eigler, Pierre, et Éric Langeard (1987), Servuction, "Le marketing des services", McGraw-Hill, Paris.

Hafsi, Taïeb, Francine Séguin, et Jean-Marie Toulouse [ $2^{e}$ éd., $3^{e}$ imp.] (2003), " La Stratégie des Organisations : une synthèse ", Les Éditions Transcontinentales, Montréal, p. 755.

Kotler, Philip, Pierre Filiatrault, et Ronald E. Turner [2 $2^{e}$ éd.] (2000), Le management du marketing, Gaetan Morin éditeur, Boucherville.

Kotler, Philip, John Bowen, et James Makens (2003), Marketing for Hospitality and Tourism, Prentice Hall, New Jersey.

Pine II, B. Joseph, et James Gilmore (1998), « Welcome to the Experience Economy », Harvard Business Review, juillet-août, Boston, p. 97-105.

Reid, Robert D., et David C. Bojanic [4éd.] (2006), Hospitality Marketing Management, John Wiley \& Sons, New Jersey.

Spears, Mariam C., et Mary B. Gregoire (2004), Food Service Organizations: A Managerial and Systems Approach, Prentice Hall, New Jersey.

Sulek, Joanne M., et Rhonda L. Hensley (2004), "The Relative Importance of Food, Atmosphere, and Fairness of Wait : The Case of a Full-service Restaurant ", Cornell Hotel and Restaurant Administration Quarterly, SAGE Publications, Thousand Oaks, CA, vol. 45, n’ 3, août, p. 235-247.

Zeithmal, Valarie A., et Mary Jo Bitner [3 $3^{e}$ éd.] (2003), Services Marketing, Integrating Customer Focus Across The Firm, McGraw-Hill, New York. 